\title{
What Is the Accretion Rate in Sagittarius A*?
}

\section{Citation}

Quataert, Eliot, Ramesh Narayan, and Mark J. Reid. 1999. "What Is the Accretion Rate in Sagittarius A*?" The Astrophysical Journal 517 (2): L101-4. https://doi.org/10.1086/312035.

\section{Permanent link}

http://nrs.harvard.edu/urn-3:HUL.InstRepos:41384923

\section{Terms of Use}

This article was downloaded from Harvard University's DASH repository, and is made available under the terms and conditions applicable to Other Posted Material, as set forth at http:// nrs.harvard.edu/urn-3:HUL.InstRepos:dash.current.terms-of-use\#LAA

\section{Share Your Story}

The Harvard community has made this article openly available.

Please share how this access benefits you. Submit a story.

Accessibility 


\title{
WHAT IS THE ACCRETION RATE IN SGR A*?
}

\author{
Eliot Quataert, Ramesh Narayan, and Mark J. Reid \\ Harvard-Smithsonian Center for Astrophysics, 60 Garden St., Cambridge, MA 02138; equataert, rnarayan, \\ mreid @cfa.harvard.edu \\ Draft version April 10, 2018
}

\begin{abstract}
The radio source $\mathrm{Sgr} \mathrm{A}^{*}$ at the center of our Galaxy is believed to be a $2.6 \times 10^{6} M_{\odot}$ black hole which accretes gas from the winds of nearby stars. We show that limits on the X-ray and infrared emission from the Galactic Center provide an upper limit of $\sim 8 \times 10^{-5} M_{\odot} \mathrm{yr}^{-1}$ on the mass accretion rate in Sgr $\mathrm{A}^{*}$. The advection-dominated accretion flow (ADAF) model favors a rate $\lesssim 10^{-5} M_{\odot} \mathrm{yr}^{-1}$. In comparison, the Bondi accretion rate onto Sgr A*, estimated using the observed spatial distribution of mass losing stars and assuming non-interacting stellar winds, is $\sim 3 \times 10^{-5} M_{\odot} \mathrm{yr}^{-1}$. There is thus rough agreement between the Bondi, the ADAF, and the X-ray inferred accretion rates for Sgr $\mathrm{A}^{*}$. We discuss uncertainties in these estimates, emphasizing the importance of upcoming observations by the Chandra $\mathrm{X}$-ray observatory $(\mathrm{CXO})$ for tightening the X-ray derived limits.
\end{abstract}

Subject Headings: accretion, accretion disks - black holes - Galaxy: center - radio sources: Sgr A*

\section{INTRODUCTION}

Stellar kinematics show that there are $\approx 2.6 \times 10^{6} M_{\odot}$ within $\approx 0.015$ pc of the Galactic Center (Eckart \& Genzel 1997, Ghez et al. 1998), centered on the radio source Sgr A* (Menten et al. 1997). The most plausible explanation is that $\mathrm{Sgr} \mathrm{A}^{*}$ is $\mathrm{a} \approx 2.6 \times 10^{6} M_{\odot}$ accreting black hole.

Sgr $A^{*}$ is believed to accrete the winds from nearby $(\sim 0.1 \mathrm{pc})$ massive stars (Krabbe et al. 1991). Hydrodynamical simulations of the Galactic Center region, assuming 10 randomly distributed point sources (to mimic the nearby stars) and a $10^{6} M_{\odot}$ black hole, yield accretion rates $\approx 1-2 \times 10^{-4} M_{\odot} \mathrm{yr}^{-1}$ (Coker \& Melia 1997; hereafter CM). If we naively increase this by a (Bondi capture) factor of $2.6^{2}$ to account for the actual mass of the central object, the theoretically predicted accretion rate becomes $\sim 10^{-3} M_{\odot} \mathrm{yr}^{-1}$ (but see $\S 3$ ).

The bolometric luminosity of $\mathrm{Sgr} \mathrm{A}^{*}$ is observed to be $\lesssim 10^{37} \operatorname{ergs~s}^{-1}$ (Genzel et al. 1994, Narayan et al. 1998). For an accretion rate of $\sim 10^{-3} M_{\odot} \mathrm{yr}^{-1}$, this corresponds to a radiative efficiency of $\sim 10^{-7}$ ! A possible explanation for the low luminosity of $\operatorname{Sgr} \mathrm{A}^{*}$ is that the accretion occurs via an advection-dominated accretion flow (ADAF; Narayan, Yi \& Mahadevan 1995, Manmoto et al. 1997, Narayan et al. 1998; see, e.g., Melia 1992, 1994, Mastichiadis \& Ozernoy 1994, Falcke 1996, Beckert \& Duschl 1997 for alternative models of Sgr A*). All ADAF models in the literature, however, require an accretion rate of $\lesssim 10^{-5} M_{\odot} \mathrm{yr}^{-1}$ (see Table 2 of Quataert \& Narayan 1999, which gives accretion rates in Eddington units; their estimates need to be multiplied by a factor of 0.055 for $\left.M_{\odot} \mathrm{yr}^{-1}\right)$. If CM's black hole mass scaled Bondi capture estimate of $\sim 10^{-3} M_{\odot} \mathrm{yr}^{-1}$ is taken at face value, there is a discrepancy of a factor of $\gtrsim 10^{2}$ in mass accretion rates.

In $\S 2$, we sharpen this discrepancy by using spectral observations of the Galactic Center to constrain the accretion rate of gas at large distances from the central black hole. The argument is nearly independent of the accretion model employed. We then calculate Bondi capture estimates of accretion onto Sgr A*, explicitly using the observed spatial distribution and wind properties of stars in the Galactic Center ( 33$)$. In $\S 4 \& \S 5$ we conclude and assess the agreement between various theoretical estimates of the accretion rate in $\operatorname{Sgr} \mathrm{A}^{*}$.

\section{OBSERVATIONAL CONSTRAINTS ON $\dot{M}$}

We use X-ray and IR limits on the emission from the Galactic Center to derive constraints on the accretion rate of optically thin or thick gas onto Sgr A*. We take the distance to the Galactic Center to be $8.0 \mathrm{kpc}$. In what follows, $m_{S}$ denotes the mass of the black hole in units of $2.6 \times 10^{6} M_{\odot}, R$ denotes the physical radius in the accretion flow, $r\left(=R / 7.38 \times 10^{11} m_{S} \mathrm{~cm}\right)$ denotes the radius in Schwarzschild units, and $\dot{m}$ is the accretion rate in Eddington units, where $\dot{M}_{e d d}=0.055 m_{S} M_{\odot} \mathrm{yr}^{-1}$.

\subsection{X-ray Constraints on Optically Thin Accretion}

If the gas accreting onto $\mathrm{Sgr} \mathrm{A}^{*}$ is optically thin, it will emit X-rays by bremsstrahlung emission. The observed limits on X-ray emission from $\mathrm{Sgr} \mathrm{A}^{*}$ can therefore be used to constrain the accretion rate. The accretion rate of the gas is related to the density, $\rho$, by the continuity equation

$$
\dot{M}=4 \pi R^{2} \rho v_{f f} \eta_{H} \eta_{v}=\dot{M}_{o}\left(r / r_{o}\right)^{p}
$$

where $r_{o} \sim 10^{5}$, the "outer" radius of the flow, is roughly the Bondi capture radius of the black hole. The radial velocity of the gas is related to the free fall velocity, $v_{f f}$, by $|v|=\eta_{v} v_{f f}$ and the vertical scale height of the gas is $H=\eta_{H} R$. For a pure Bondi flow, $\eta_{v}=\eta_{H}=1$, while for an $\mathrm{ADAF}, \eta_{v} \approx \alpha$ (the viscosity parameter) and $\eta_{H} \approx 1 / 2$.

Equation (11) allows for the possibility that the mass accretion rate may decrease with radius due to an outflow/wind (in which case the assumed geometry is a "thick" equatorial inflow with a bipolar outflowing wind; cf Blandford \& Begelman 1999). The accretion rate at the 
outer edge of the flow (i.e., the mass supply rate) is $\dot{M}_{o}$ and the parameter $p$ measures the strength of the outflow.

We parameterize the electron temperature in the accretion flow by $T_{e}=\eta_{T} T_{o}\left(r / r_{o}\right)^{-a}$, where $T_{o}=10^{12} / r_{o}$ is $\sim$ the proton virial temperature at $r_{o}$ and $a(\sim 1$ at large radii) determines the radial profile of the electron temperature; $\eta_{T} \approx 1$ for both Bondi accretion and an ADAF.

The bremsstrahlung luminosity at frequency $\nu$ is given by

$$
L_{\nu}=4 \pi \eta_{H} \int \epsilon_{\nu} R^{2} d R,
$$

where $\epsilon_{\nu}=2.6 \times 10^{10} \rho^{2} T_{e}^{-1 / 2} \exp \left[-h \nu / k T_{e}\right] \operatorname{ergs~s}^{-1} \mathrm{~cm}^{-3}$ is the bremsstrahlung emissivity (e.g., Rybicki \& Lightman 1979; we have ignored a weak frequency-dependent Gaunt factor). Carrying out the integral in equation (2) using the above expressions for the density and temperature of the gas, and approximating the exponential cutoff in $\epsilon_{\nu}$ by a step function, we find (in ergs s${ }^{-1} \mathrm{~Hz}^{-1}$ )

$$
L_{\nu} \approx \frac{6 \times 10^{19}}{2 p+a / 2} \eta_{H}^{-1} \eta_{v}^{-2} \eta_{T}^{-1 / 2} \dot{m}_{o}^{2} m_{S} r_{o}^{1 / 2}\left(\frac{r_{\nu}}{r_{o}}\right)^{2 p+a / 2}
$$

where $\dot{m}_{o}=\dot{M}_{o} / \dot{M}_{e d d}$ and

$$
r_{\nu}=r_{o} \operatorname{Min}\left[1,\left(\frac{h \nu}{\eta_{T} k T_{o}}\right)^{-1 / a}\right] .
$$

Observations at frequency $\nu$ are dominated by emission from the radius $r_{\nu}$. In particular, if the X-ray energies of interest are less than the minimum electron thermal energy in the flow (which is obtained at $r_{o}$ and is $\sim 1 \mathrm{keV}$ for $r_{o} \sim 10^{5}$ ), the bremsstrahlung luminosity is dominated by emission from the largest radii in the flow. In this case $r_{\nu}=r_{o}$ and the parameters $p$ and $a$, which determine the radial profiles of the density and electron temperature, are irrelevant; they enter only in the normalization of the emission, not in the exponent of any parameters of the problem. As a result, observations of soft X-rays provide effective constraints on $\dot{M}_{o}$, the rate at which matter is supplied to the accretion flow on the outside. They cannot, however, constrain the flow structure closer to the black hole (e.g., the nature or strength of an outflow).

Parameterizing the observational constraints on emission from the Galactic Center via $\nu L_{\nu} \lesssim 10^{34} L_{34} \mathrm{ergs} \mathrm{s}^{-1}$ at $h \nu=\nu_{1} \mathrm{keV}\left(\nu=2.4 \times 10^{17} \nu_{1} \mathrm{~Hz}\right)$ equation (3) can be inverted to give a constraint on the accretion rate of the gas (taking $2 p+a / 2 \approx 1$ in the denominator of eq. [3] for simplicity):

$$
\begin{aligned}
\dot{M}_{o} & \lesssim 8 \times 10^{-5} r_{5}^{-1 / 4} \eta_{H}^{1 / 2} \eta_{v} \eta_{T}^{1 / 4} m_{S}^{1 / 2} \\
& \times\left(\frac{L_{34}}{\nu_{1}}\right)^{1 / 2}\left(\frac{r_{o}}{r_{\nu}}\right)^{p+a / 4} \mathrm{M}_{\odot} \mathrm{yr}^{-1},
\end{aligned}
$$

where $r_{5}=r_{o} / 10^{5}$.

The parameters $\eta_{v}, \eta_{H}$, and $\eta_{T}$ must all be $<1$. Furthermore, the Bondi capture radius in Sgr $\mathrm{A}^{*}$ for wind material moving at $\sim 10^{3} \mathrm{~km} \mathrm{~s}^{-1}$ is $\sim 10^{5}$ Schwarzschild radii so that the accretion flow must originate at $r_{5} \sim 1$.

ROSAT observations of the Galactic Center indicate that the X-ray luminosity of Sgr $\mathrm{A}^{*}$ at $\approx 1 \mathrm{keV}$ is $\lesssim 10^{34}$ ergs $\mathrm{s}^{-1}$, in which case $L_{34} \approx \nu_{1} \approx 1$ and $r_{\nu} \approx r_{o}$ (but, see Narayan et al. 1998 for a discussion of the effect of uncertainties in the absorbing column; we take $N_{H}=6 \times 10^{22} \mathrm{~cm}^{-2}$ ). By equation (5) this implies $\dot{M}_{o} \lesssim 8 \times 10^{-5} \mathrm{M}_{\odot} \mathrm{yr}^{-1}$; this is a strict upper limit on the accretion rate of optically thin gas in Sgr A*.

ASCA observations of the Galactic Center (Koyama et al. 1996) give a $2-10 \mathrm{keV}$ limit of $10^{35} \mathrm{ergs} \mathrm{s}^{-1}$. Taking $\nu_{1} \approx 5$, equation (河) gives a limit of $\dot{M}_{\nu} \lesssim 10^{-4} M_{\odot} \mathrm{yr}^{-1}$ at $r_{\nu} \approx 0.2 r_{o} \sim 10^{4}$, where $\dot{M}_{\nu}=\dot{M}_{o}\left(r_{\nu} / r_{o}\right)^{p}$ is the accretion rate at the radius $r_{\nu} \approx r_{o} \nu_{1}^{-1 / a} \eta_{T}^{1 / a}$. SIGMA observations (Vargas et al. 1998) give $\nu L_{\nu} \lesssim 2 \times 10^{35}$ ergs $\mathrm{s}^{-1}$ at $\nu_{1} \approx 75$, which requires $\dot{M}_{\nu} \lesssim 10^{-4} M_{\odot} \mathrm{yr}^{-1}$ at $r_{\nu} \approx 0.01 r_{o} \sim 10^{3}$. If there are no winds emanating from Sgr $\mathrm{A}^{*}$ at $r \sim r_{0}$ (i.e., $p \approx 0$ ) then the ASCA and SIGMA observations require $\dot{M}_{o} \lesssim 10^{-4} M_{\odot} \mathrm{yr}^{-1}$. This may be an even stronger limit than that derived from the ROSAT observations, since it is insensitive to the absorbing column.

\subsection{Infrared Constraints on Optically Thick Accretion}

The preceding subsection assumes that the gas is optically thin at large radii. We believe that this is the most likely scenario. It is worth, however, examining observational constraints imposed on the accretion rate of an optically thick disk.

For a standard Shakura-Sunyaev disk (e.g., Frank, King, \& Raine 1992), the luminosity is given by

$$
\nu L_{\nu}=\frac{16 \pi^{2} h \cos (i) \nu^{4}}{c^{2}} \int_{R_{\text {in }}}^{R_{\text {out }}} \frac{R d R}{\exp (h \nu / k T(R))-1},
$$

where $i$ is the inclination of the disk, $R_{\text {out }}$ and $R_{\text {in }}$ are the inner and outer radii of the disk, and $T(R)$, the effective temperature of the disk, depends on the accretion rate ( $\mathrm{cf}$ Frank et al. 1992).

Menten et al. (1997) have obtained an upper limit of $\approx 10^{35} \mathrm{ergs} \mathrm{s}^{-1}$ on the $2.2 \mu \mathrm{m}$ emission from the Galactic Center. This emission strongly constrains the properties of any geometrically thin disk. It is straightforward to numerically integrate equation (6) and obtain the $2.2 \mu$ emission from the disk. Requiring this to be $\lesssim 10^{35}$ ergs $\mathrm{s}^{-1}$ yields a constraint on the parameters $\dot{M}$ and $r_{i n}$. For $i \sim 60^{\circ}$, taking $\dot{M} \approx 10^{-3} \mathrm{M}_{\odot} \mathrm{yr}^{-1}$ implies $r_{i n} \gtrsim 10^{4}$. If $\dot{M} \approx 10^{-5} \mathrm{M}_{\odot} \mathrm{yr}^{-1}$ the constraint is slightly less stringent, $r_{\text {in }} \gtrsim 3 \times 10^{3}$ (note that, to satisfy all existing IR and radio limits on emission from Sgr $\mathrm{A}^{*}$ typically requires even larger values of $\left.r_{i n}\right){ }^{1}$

This implies that, for any reasonable inclination and accretion rate, a thin disk cannot extend much inside $r_{\text {in }} \sim 10^{4}$ without violating the IR limits on emission from the Galactic center. Even if we assume that from the Bondi capture radius to $r_{i n}$ the accretion is via a geometrically thin disk, and then for $r<r_{i n}$ it is optically thin, our limits on $\dot{M}$ from the previous subsection are

\footnotetext{
${ }^{1}$ Genzel et al. (1997) report a possible detection of Sgr $\mathrm{A}^{*}$ at $2.2 \mu \mathrm{m}$, at a flux level above Menten et al.'s upper limit (indicating possible variability in the source). This need not represent a detection of an optically thick disk, but could be either (1) singly Compton scattered synchrotron emission, (2) synchrotron emission from non-thermal electrons, or (3) optically thick accretion disk emission. If interpreted as the latter, the upper limits in this paragraph become (approximate) equalities.
} 
not changed substantially. This is because the X-ray constraints on the accretion rate are quite insensitive to the precise outer boundary of the optically thin region (cf. eq. [5] where the limit is $\left.\propto r_{o}^{-1 / 4}\right)$.

\section{BONDI CAPTURE ESTIMATES}

There are $\approx 10$ He I stars (Krabbe et al. 1991) projected within 10 arcsec of the position of Sgr A* (Menten et al. 1997). The winds from these stars result in mass loss rates ranging from about $8 \times 10^{-5}$ to $8 \times 10^{-4} M_{\odot} \mathrm{yr}^{-1}$ and might provide most of the matter accreted by Sgr A*

Using the distribution of the He I stars projected on the plane of the sky, their mass loss rates, $\dot{M}_{w}$, and their wind speeds, $V_{w}$ (Najarro et al. 1997), we have estimated the mass accretion rate onto a $2.6 \times 10^{6} M_{\odot}$ black hole. The line-of-sight (z-coordinate) offset of each star relative to Sgr A* (the black hole) is unknown, and we assigned a z-distance randomly to each star, assuming a Gaussian distribution. The stellar wind material passing within a radius $R_{A}=2 G M_{B H} / V_{w}^{2}$ of $\mathrm{Sgr} \mathrm{A}^{*}$ was assumed to be accreted. This resulted in a contribution to the accretion rate for a given star, $\dot{M}_{a c c}$, given by

$$
\dot{M}_{a c c}=\frac{1}{2} \dot{M}_{w}\left(1-\sqrt{1-R_{A}^{2} / r^{2}}\right),
$$

where $r$ is the three-dimensional distance of the star from Sgr $A^{*}$ (Note that all stars in the Najarro et al. sample have $\left.r>R_{A}\right)$.

Using equation (7), a star such as IRS $16 \mathrm{C}$ with $\dot{M}_{w} \approx$ $8 \times 10^{-5} M_{\odot} \mathrm{yr}^{-1}$, a wind speed of $650 \mathrm{~km} \mathrm{~s}^{-1}$, and a true distance from $\mathrm{Sgr} \mathrm{A}^{*}$ of $\approx 2$ arcsec (its projected distance is $1.4 \mathrm{arcsec}$ ) would contribute about $1.4 \times 10^{-5} M_{\odot} \mathrm{yr}^{-1}$ to the total mass accretion rate of $\mathrm{Sgr} \mathrm{A}^{*}$. On the other hand, the most powerful He I star near Sgr A*, IRS 13E1, which loses mass at a rate of $8 \times 10^{-4} M_{\odot} \mathrm{yr}^{-1}$ with a wind speed of $1000 \mathrm{~km} \mathrm{~s}^{-1}$, has a projected distance from Sgr $\mathrm{A}^{*}$ of 3.7 arcsec and thus makes a maximum contribution to the mass accretion rate of only $5 \times 10^{-6} M_{\odot} \mathrm{yr}^{-1}$.

For each trial distribution of stars, obtained for different realizations of the random z-coordinate distances from Sgr $A^{*}$, we calculated the total mass accretion rate as the sum of the accretion rates for each star. This assumes no wind-wind interactions. Varying the standard deviation of the z-coordinate distribution, $\sigma_{z}$, we found average total mass accretion rates of $\dot{M}_{o}=5,3$, and $2 \times 10^{-5} M_{\odot} \mathrm{yr}^{-1}$ for $\sigma_{z}$ of 2,5 , and 8 arcseconds, respectively. ${ }^{2}$ If the $\mathrm{He}$ I stars have a $\sigma_{z} \approx 5$, as suggested by the projected spatial distribution of stars near Sgr A* (Genzel et al. 1997; Ghez et al. 1998), then the total mass accretion rate for non-interacting stellar winds onto a $2.6 \times 10^{6} M_{\odot}$ black hole should be $\dot{M}_{o} \approx 3 \times 10^{-5} M_{\odot} \mathrm{yr}^{-1}$.

This accretion rate is noticeably smaller than the value of $\sim 10^{-3} M_{\odot} \mathrm{yr}^{-1}$ obtained by scaling CM's numerical results from a $10^{6} M_{\odot}$ black hole to a $2.6 \times 10^{6} M_{\odot}$ black hole (cf. §1). The main reason for the discrepancy seems to be that the spatial distribution of point sources they take (e.g., $\pm 3 R_{A}$ ) is a reasonable approximation of the observed stellar distribution only for a $2.6 \times 10^{6} M_{\odot}$ black hole, not a $10^{6} M_{\odot}$ hole. That is, for $v_{w} \approx 700 \mathrm{~km} \mathrm{~s}^{-1}$ and a black hole mass of $10^{6} M_{\odot}, 3 R_{A}$ is only $\approx 1^{\prime \prime}$, which is smaller than the typical projected distance of the mass losing stars from Sgr A* (on the other hand, for a $2.6 \times 10^{6} M_{\odot}$ black hole, $3 R_{A}$ corresponds to $\left.\approx 3^{\prime \prime}\right)$. One should therefore not scale CM's result up by a factor of $2.6^{2}$. This leaves a smaller residual discrepancy, perhaps due to wind-wind interactions, which they treat in their hydrodynamical simulations, but which we neglect.

\section{DISCUSSION}

In $\S 2$ of this paper, we showed that ROSAT X-ray observations of Sgr $\mathrm{A}^{*}$ provide a firm limit on the accretion rate at large radii, namely $\dot{M}_{o} \lesssim 8 \times 10^{-5} \eta_{v} L_{34}^{1 / 2} M_{\odot} \mathrm{yr}^{-1}$ at $r \approx 10^{5}$, where $\eta_{v}$ is the ratio of the radial velocity of the gas to the free fall velocity and $L_{34}$ is the soft X-ray $(\approx 1 \mathrm{keV})$ luminosity of the source. We obtain similar limits at $r \sim 10^{4}$ and $r \sim 10^{3}$ using ASCA and SIGMA data, respectively. It is important to emphasize that these limits correspond to the X-ray radiation being dominated by bremsstrahlung emission. If part of the $\mathrm{X}$-rays are produced by Comptonization, as in some models, then the accretion rates must be even lower than the limits derived here.

The X-ray luminosity of $10^{34} \mathrm{erg} \mathrm{s}^{-1}$ used in the above scalings is itself an upper limit. There is considerable diffuse emission from the Galactic Center (Koyama et al. 1996), and the luminosity of the accretion flow is uncertain. Another uncertainty is the absorbing column, which could lie anywhere from the value used here, $6 \times 10^{22} \mathrm{~cm}^{-2}$ (Watson et al. 1981), to $1.5 \times 10^{23} \mathrm{~cm}^{-2}$ (Predehl \& Trumper 1994). If the column is closer to the higher value, then the unabsorbed luminosity of Sgr A* could be larger than we have assumed. High angular resolution observations with CXO (formerly AXAF) should solve both problems.

Sgr A* probably accretes most of its mass from the winds of nearby massive stars (Krabbe et al. 1991). We have estimated the Bondi accretion rate onto Sgr $\mathrm{A}^{*}$ using the observed spatial distribution of these stars, along with their estimated mass loss rates and outflow velocities (§3). The resulting accretion rate is $\dot{M}_{o} \approx 3 \times 10^{-5} M_{\odot} \mathrm{yr}^{-1}$ for plausible values of the unknown spatial distribution of stars along the line of sight. This estimate of $\dot{M}_{o}$ should perhaps be interpreted as a lower limit, as there may be other sources of matter for the accretion flow, and windwind interactions and post-shock cooling may increase the fraction of the wind material accreted.

The upper limit on $\dot{M}_{o}$ from the X-ray observations and the lower limit on $\dot{M}_{o}$ from the capture of stellar winds are roughly compatible, requiring $\eta_{v} \sim 1$ for the accretion flow and $L_{34} \sim 1$ for the (unknown) X-ray luminosity. If the accretion onto $\mathrm{Sgr} \mathrm{A}^{*}$ occurs viscously via an ADAF, then $\eta_{v} \approx \alpha$. For values of $\alpha \approx 0.01$ seen in numerical simulations of thin disks (Hawley, Gammie, \& Balbus, 1996), there is a substantial discrepancy between the Bondi and X-ray inferred accretion rates, while for the values of $\alpha \approx 0.1-0.3$ favored by Narayan et al. (1998; see also Esin et al. 1997), the two estimates are comparable.

Blandford \& Begelman (1999) have recently argued that only a small fraction of the mass supplied to an ADAF reaches the central black hole, with most of it being lost to an outflow/wind. It is important to emphasize that this

${ }^{2}$ For an "average" wind speed of $700 v_{7} \mathrm{~km} \mathrm{~s}^{-1}$, this corresponds to $\sigma_{z}$ of $2,5, \& 8 v_{7}^{-2} R_{A}$. 
alone will not modify the conclusions of this paper. Our primary constraint on the accretion rate utilizes the $\sim 1$ $\mathrm{keV}$ X-ray flux, which is dominated by bremsstrahlung emission from large radii in the flow $\left(r \approx r_{0} \approx 10^{5}\right)$; at such radii, any wind from the accretion flow has little effect.

Gruzinov (1999) has argued that radial heat conduction would significantly reduce the mass accretion rate in Bondi flows. This explanation is also unlikely to effect the results of this paper. The density at $\sim r_{0}$ is a boundary condition set by the stellar winds. Gruzinov's flow would therefore produce the same X-ray luminosity from radii $r \sim r_{0}$ as the models we have considered (at large radii $\sim r_{0}$, Gruzinov's reduced accretion rate is due to a smaller radial velocity, not a lower density).

Finally, it is worth emphasizing that our paper does not explicitly address the reason for the low radiative efficiency of Sgr $\mathrm{A}^{*}$. This is determined by the physical conditions close to the black hole $(r \lesssim 10-100)$, while our analysis focuses on larger radii.

\section{CONCLUSION}

With current data, we believe that there is rough agreement between the Bondi accretion rate (as estimated in this paper), the limits inferred from X-ray and IR observations, and the value (or, more precisely, the upper limit) favored by ADAF models. If this straightforward interpretation is correct, CXO should detect an X-ray flux comparable to that of ROSAT, despite its significantly improved angular resolution. On the other hand, if CXO substantially lowers the soft X-ray flux from the Galactic Center there would be an inconsistency between the Bondi rate and the values favored by the X-ray observations. Given the uncertainties in the accretion rate estimates, it is worth spelling out what we believe to be the two plausible, if somewhat mundane, explanations if such an inconsistency is discovered.
The first assumes that the Bondi accretion rate estimates are correct and $\dot{M}_{o} \gtrsim 3 \times 10^{-5} M_{\odot} \mathrm{yr}^{-1}$. This could be reconciled with the limits inferred from a significantly smaller soft X-ray flux only if $N_{H}$ is larger, by a factor of few, than the value we have assumed $\left(6 \times 10^{22} \mathrm{~cm}^{-2}\right)$.

The other alternative is simply that the Bondi accretion rate estimates at large radii are in error, with the true accretion rate being $\lesssim 10^{-5} M_{\odot} \mathrm{yr}^{-1}$. We see several possible reasons for such an error:

1. The estimates of the stellar mass loss parameters, $\dot{M}_{w}$ and $V_{w}$, may be in error. Note that a $25 \%$ uncertainty in $V_{w}$ translates to a factor of 2.5 uncertainty in the Bondi accretion rate. Together with a factor of few uncertainty in the stellar mass loss rates, $\dot{M}_{w}$, the Bondi accretion rate is probably uncertain by at least a factor of $\sim 5$.

2. If the stars in the Galactic Center are not randomly distributed around Sgr $\mathrm{A}^{*}$, but instead have a large $\mathrm{z}$ coordinate offset, the predicted accretion rate will be reduced. We find that a systematic offset of $\gtrsim 0.5 \mathrm{pc}$ along the line of sight is needed to bring $\dot{M}_{o} \lesssim 10^{-5} M_{\odot} \mathrm{yr}^{-1}$.

3. Perhaps Sgr $\mathrm{A}^{*}$ has a strong outward wind which maintains a low-density "bubble" around the source (the winds from the stars in the small stellar cluster within 1" of Sgr A* - possibly 09 stars; Genzel et al. 1997 - might also contribute sufficient momentum to partially impede accretion of the HeI stars winds). If this bubble extends beyond the accretion radius $R_{A}$, then the accretion rate could be significantly reduced. The bubble is likely to be held up by mechanical pressure from a wind rather than radiative heating since the luminosity of $\mathrm{Sgr} \mathrm{A}^{*}$ is much too low for the latter to be efficient.

We thank Rob Coker for useful conversations and the referee for useful comments. We acknowledge support from an NSF Graduate Research Fellowship (E.Q.) and NAG 5-2837 (R.N.).

\section{REFERENCES}

Beckert, T., \& Duschl, W. J. 1997, A\&A, 328, 95

R D. \& Begelman, M. C., 1999, MNRAS in press astro$\mathrm{ph} / 9809083$

Coker, R. \& Melia, F., 1997, ApJ, 488, L14 (CM)

Eckart, A., \& Genzel, R. 1997, MNRAS, 284, 576

Esin, A. A., McClintock, J. E., \& Narayan, R., 1997, ApJ, 489, 86

Falcke, H., 1996, in IAU Symp. 169, Unsolved Problems of the Milky

Way, eds. L. Blitz \& P. J. Teuben (Dordrecht: Kluwer), 163

Frank, J., King, A., \& Raine, D. 1992, Accretion Power in Astrophysics, (Cambridge: Cambridge Univ. Press

Genzel, R., Eckart, A., Ott, T., \& Eisenhauer, F., 1997, MNRAS, 291, 219

Genzel, R., Hollenbach, D., \& Townes, C. H. 1994, Rep Prog. Phys., 57,417

Ghez, A. M., Klein, B. L., Morris, M., \& Becklin, E. E., 1998, ApJ, 509, 678

Gruzinov, A., 1999, ApJ in press astro-ph/9809265

Hawley, J. F., Gammie, C. F., \& Balbus, S. A., 1996, ApJ, 464, 690

Koyama, K. et al. 1996, PASJ, 48, 249
Krabbe, A., Genzel, R., Drapatz, S. \& Rotaciuc, V., 1991, ApJ, 382, L19

Manmoto, T., Mineshige, S., \& Kusunose, M., 1997, ApJ, 489, 791

Mastichiadis, A., \& Ozernoy, L. M., 1994, ApJ, 426, 599-603

Melia, F., 1992, ApJ, 387, L25

Melia, F., 1994, ApJ, 426, 577

Menten, K. M., Reid, M. J., Eckart, A., \& Genzel, R. 1997, ApJ, 475, L111

Najarro, F. et al. 1997, A \& A, 325, 700

Narayan, R., Mahadevan, R., Grindlay, J.E., Popham, R.G., \& Gammie, C., 1998a, ApJ, 492, 55

Narayan, R., Yi, I., \& Mahadevan, R., 1995, Nature, 374, 623

Predehl, P., \& Trumper, J. 1994, A\&A, 290, L29

Quataert, E. \& Narayan, R., 1999, ApJ in press astro-ph/9810136)

Rybicki, G. \& Lightman, A., 1979, Radiattve Processes in Astrophysics (New York: John Wiley \& Sons

Vargas. M. et al., 1996, in The Galactic Center, ed. R. Gredel, 431

Watson, M. G., Willingale, R., Grindlay, J. E., \& Hertz, P., 1981, ApJ, 250, 142 